\title{
Optical Analysis of Complex Multilayer Structures Using Multiple Data Types
}

\author{
Blaine Johs $^{*}$, Roger H. French ${ }^{+}$, Franklin D. Kalk ${ }^{++}$, William A. McGahan ${ }^{*}$, and John A. Woollam ${ }^{*}$ \\ *.A. Woollam Co., Inc., 650 J. Street, Suite 39, Lincoln, NE 68508 USA \\ DuPont Co. ${ }^{+}$Central Research and ${ }^{++}$Electronic Materials, Wilmington, DE 19880-0356 USA
}

\begin{abstract}
$\underline{\text { ABSTRACT }}$
Variable angle of incidence spectroscopic ellipsometry (VASE) is commonly used for multilayer optical analysis, but in some cases this experiment (performed in reflection) does not provide sufficient information for the unique determination of the thicknesses and optical constants of the films in the given multilayer. We have found that augmenting the VASE data with data from other optical experiments greatly increases the amount of information which can be obtained for multilayers, particularly when they are deposited on transparent substrates. In this work, we describe a formalism which allows us to quantitatively characterize complex multilayer structures by using combined reflection and transmission ellipsometry, reflection ellipsometry with the sample flipped over, and intensity transmission measurements. To demonstrate the usefulness of this capability, the analysis of a complex graded, absorbing thin film structure (a Cr-based phase-shifting photomask blank), is presented.
\end{abstract}

\section{INTRODUCTION}

Variable angle of incidence spectroscopic ellipsometry (VASE) is a powerful tool for multilayer optical analysis. On an 'ideal' multilayer sample (a sample where the layer thicknesses are approximately greater than $\lambda / 4$ of the measurement beam and the films are transparent), VASE can simultaneously determine multiple layer thicknesses and optical constants. However, if the films are absorbing, it can be difficult to uniquely extract the film thickness ' $t$ ', index ' $n$ ', and extinction coefficient ' $\mathrm{k}$ ' from VASE data alone. It has been shown that combining VASE data with intensity transmission data is an

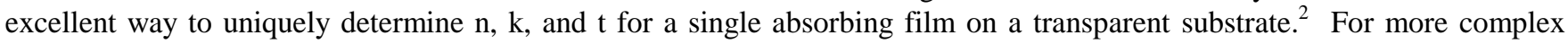
multilayer structures, even more optical information is necessary to uniquely determine multiple thicknesses and the optical constants of more than one absorbing film. Section 2 outlines the procedure for combining multiple optical data types (VASE, intensity transmission, ellipsometry with backside reflections, ellipsometry with the sample flipped, and transmission ellipsometry) for characterization of complex multilayer structures.

Traditionally, ellipsometric measurements of films on thick transparent substrates have been complicated by the incoherent reflections from the backside of the substrate; removal of the backside reflections by roughening the backside of the substrate or spatially filtering the backside reflections from the detector was required to obtain accurate ellipsometric data that could be reliably interpreted. In section 3, a formalism is presented that accurately describes the effect of the incoherent reflections on the data acquired by a rotating element ellipsometer (which can be a rotating analyzer, RAE, or rotating polarizer, RPE, configuration). Using this formalism, it is possible to quantitatively model the ellipsometric data acquired for films on thick transparent substrates in the following configurations: 1) standard VASE data (acquired with the backside of the substrate roughened to suppress backreflections), 2) reflection ellipsometric data, including the incoherent backside reflections, 3) reflection ellipsometric data with the sample 'flipped' or turned over, such that the input beam is incident on the opposite side of the sample, and 4) transmission ellipsometric data, including the incoherent transmitted beams.

A real-world example that illustrates the utility of multiple optical data type analysis is shown in section 4 . The analysis of a Cr-based phase-shifting photomask blank is presented. In this complex thin film structure, the composition of a Cr-based absorbing material is intentionally graded during the deposition process. Multiple optical data types were used to uniquely extract the film thickness, index gradient, and optical constants of the two absorbing constituent materials in the complex thin film structure. The final optical model for the sample accurately predicted the transmitted phase shift relative to air for the sample, which was independently measured by a laser based dual beam phase interferometer. The structural parameters determined in the optical model, namely the film thickness and index gradient profile, were also independently confirmed by stylus profilometry, Rutherford Backscattering Spectrometry (RBS), and Sputtered Neutrals Mass Spectrometry (SNMS).

B. Johs, R. H. French, F. D. Kalk, W. A. McGahan, J. A. Woollam, "Optical Analysis of Complex Multilayer Structures Using Multiple Data Types", Optical Interference Coatings, SPIE Vol. 2253, Edited by F. Abeles, 1098-1106, (1994). 


\section{MODEL-BASED ANALYSIS WITH MULTIPLE OPTICAL DATA TYPES}

To extract physical sample parameters (such as film thicknesses, optical constants, etc.) from experimentally measured optical data, a model-based analysis procedure is used. An optical model is constructed by sequentially adding layers on top of a substrate material. Each layer is parameterized by a thickness and complex index of refraction. All layers are also assumed to be homogeneous, with abrupt and parallel interfaces. Using formulas for the Fresnel reflection coefficients, Snell's law, and other thin film optics equations, the complex amplitude reflectivity (and transmissivity) of the layered stack can be calculated for both p-polarized and s-polarized light (denoted $R_{p}$ and $R_{s}, T_{p}$ and $T_{s}$ ), at any given angle of incidence and wavelength. Ellipsometry measures the complex ratio of the $\mathrm{p}$ - and s-components, which is traditionally defined as $\tan (\Psi) \mathrm{e}^{\Delta \cdot \mathrm{i}}=\mathrm{R}_{\mathrm{p}} / \mathrm{R}_{\mathrm{s}}$ (for transmission ellipsometry, the definition becomes: $\tan (\Psi) \mathrm{e}^{\Delta \cdot \mathrm{i}}=\mathrm{T}_{\mathrm{p}} / \mathrm{T}_{\mathrm{s}}$ ). In a similar manner, the optical model may also be used to calculate the reflected and transmitted light intensity. For example, the reflected intensity for p-polarized light is given by $\left(\left|R_{p}\right|\right)^{2}$.

Given the ability to calculate predicted $\Psi, \Delta$, reflectivity, transmission, etc. values for a given model, we can now compare the model predicted values with experimental values from the corresponding optical measurement. The difference between the model calculated and experimental data is objectively quantified by equation (1), which defines a figure of merit function denoted as the weighted root Mean Squared Error (MSE).

$$
M S E=\sqrt{\frac{1}{N-M} \sum_{i=1}^{N}\left(\frac{\text { ModelCalc(type }, \phi, \lambda, \text { Parms }[M])- \text { ExpData }_{i}}{\sigma_{i}}\right)^{2}}
$$

In this equation, there are ' $\mathrm{N}$ ' experimental measurements, labeled as ExpData $\mathrm{i}_{\mathrm{i}}$. Each experimental measurement has an uncertainty $\sigma_{\mathrm{i}}$ (or standard deviation) associated with it, which weights the measurement relative to the other experimental data points. The model is calculated using the corresponding optical data type, angle of incidence $\phi$, and wavelength $\lambda$ at each experimental data point. The model calculation is also a function of ' $\mathrm{M}$ ' adjustable, or 'fit', parameters.

Equation (1) is very general, in that it can handle any type or combination of optical data types that can be calculated by the model. If the model calculated optical data agrees with the experimentally measured optical data, the MSE will be low, and the model parameters (layer thicknesses, optical constants, etc.) may represent the actual physical parameters of the sample. If the model calculated data does not agree with the experimentally measured data, the MSE will be higher, and the model parameters should be adjusted to minimize the MSE; this process is know as 'fitting' the model to the experimental data. Fitting the model to the experimental data is not trivial, as there may be many (>200) model parameters, and the model calculation is in general non-linear with respect to its parameters. A suitable fitting algorithm is the Levenberg-Marquardt method, ${ }^{4}$ which iteratively adjusts the model parameters to minimize the MSE. All of the optical model calculations and subsequent data fits in this work were performed using the WVASE optical modeling and analysis software.

\section{CALCULATION OF ELLIPSOMETRIC $\Psi$ AND $\triangle$ WITH INCOHERENT REFLECTIONS}

Consider the optical measurement beam configuration shown in figure 1. In this figure, the input beam is incident on the front side (film side) of the sample. The beam propagates coherently through the multilayer film stack, resulting in a directly reflected beam (Rf) and a transmitted beam (Tf). Rf and $\mathrm{Tf}$ (along with the other Fresnel reflection and transmission coefficients labeled in figure 1) are readily calculated using the optical model formalism described in section 2 . The transmitted beam incoherently propagates through the thick substrate; part of this beam is then reflected by the backside substrate/air interface, which in turn may be reflected from the substrate/film interface, etc. The multiple internal substrate reflections result in multiple reflected and transmitted beams. The separation of these beams is a function of the substrate thickness and index of refraction, and the input beam angle of incidence. As the multiple reflected and transmitted beams are incoherent with each other, the beam intensities may be simply added together without considering interference effects, which after simplifying the convergent infinite series leads to the well known expressions ${ }^{6}$ shown in equations (2) and (3). These expressions represent the total reflected and transmitted beam intensities when all of the incoherent beams are collected by the detector. 


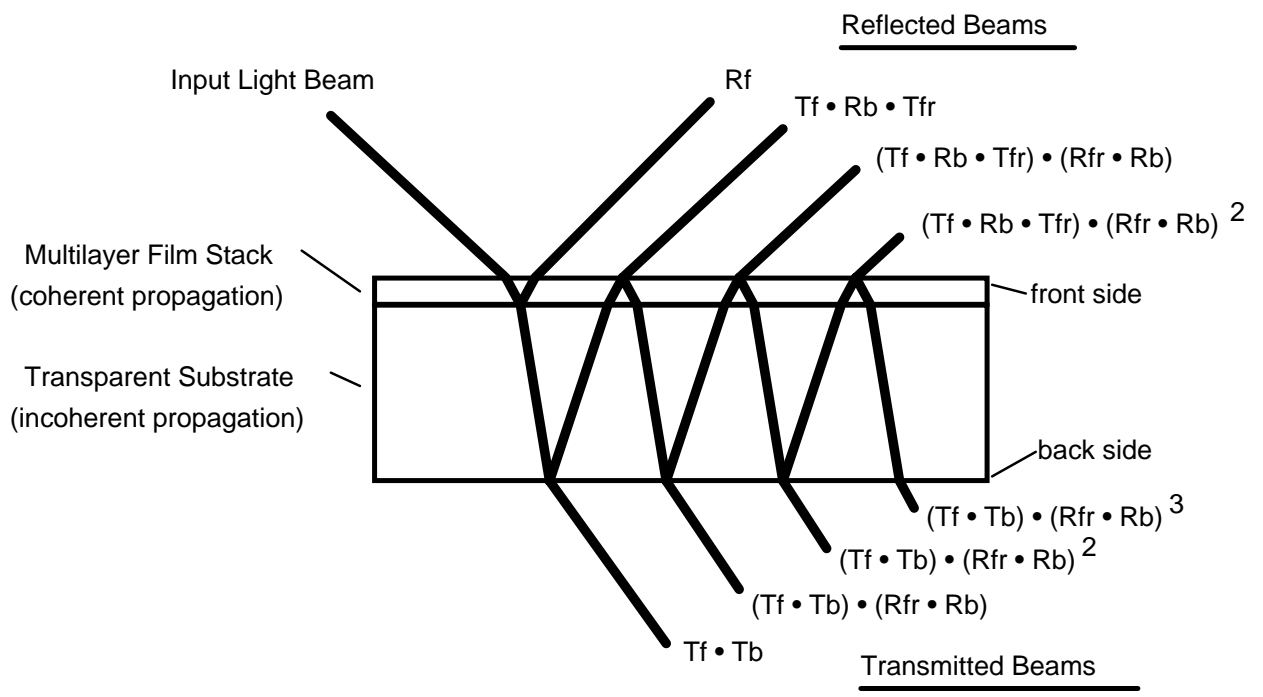

Fresnel Coefficient Definitions (complex field amplitudes)

$\mathrm{Rf}$ ambient/film stack reflection

Tf film stack/substrate transmission

$\mathrm{Rb}$ substrate/ambient reflection

Tb substrate/ambient transmission

Rfr substrate/film stack reflection

Tfr film stack/ambient transmission

Figure 1. Optical measurement beam configuration on transparent substrate illustrating multiple incoherent reflections.

$$
\mathrm{R}_{\text {all }}=|R f|^{2}+\frac{|T f|^{2} \cdot|R b|^{2} \cdot|T f r|^{2}}{1-|R f r|^{2} \cdot|R b|^{2}} \quad \text { (2) } \quad \mathrm{T}_{\text {all }}=\frac{|T f|^{2} \cdot|T b r|^{2}}{1-|R f r|^{2} \cdot|R b|^{2}}
$$

The 'effective' (or measured) ellipsometric $\Psi$ parameter for the multiple reflected beams may be calculated by applying equations (2) and (3) for both p- and s- polarized beams. However, as only the beam intensities were used in this derivation, the phase information required to calculate the ellipsometric $\Delta$ parameter is not available. To calculate the 'effective' $\Delta$ measured by an ellipsometer in the presence of multiple incoherent beams, it is important to realize that while the absolute phase of the propagating beam is unknown after it incoherently traverses the thick substrate, the relative phase between the p-and s- components of the beam is maintained, even though the wave is spatially incoherent across the substrate. Another way of stating this is that the polarization state of the beam remains unchanged as it incoherently propagates through the substrate (assuming the substrate is optically isotropic). If the substrate is absorbing, both p- and scomponents of the beam will be equally attenuated, but the relative p- to s- phase difference will not change. Therefore, it is possible to exactly calculate the ratio of p- to s- field intensities $(\tan \Psi)$ and the phase difference between p- and s- beam components $(\Delta)$ for each individual reflected and transmitted beam by evaluating the expressions in figure 1 for both $\mathrm{p}$ - and spolarizations.

If it is possible for the ellipsometer's detector system to collect only a single reflected or transmitted beam, the expressions given in figure 1 can accurately predict the measured ellipsometric data. However, for many samples the substrate thickness and measurement beam size are such that it is not possible to separate out the multiple beams; for example, the ellipsometer detector system may collect $1.5,2,3.88$ or all of the multiple beams. In this case, the ellipsometer detector system no longer measures a totally polarized beam, but a partially polarized beam. The traditional definition of the ellipsometric $\Psi$ and $\Delta$ values given in section 2 is not valid when the sample partially polarizes the measurement beam. The ellipsometer system, however, will still measure 'effective' ellipsometric parameters for the partially polarized beam, which we denote as $\Psi_{\text {eff }}$ and $\Delta_{\text {eff. }}$. We next consider how to accurately calculate the $\Psi_{\text {eff }}$ and $\Delta_{\text {eff }}$ for the rotating element ellipsometer configuration. 
The signal intensity measured by a rotating element ellipsometer detector is given in equation (4) which is valid for both the rotating analyzer configuration (RAE) or the rotating polarizer configuration (RPE). In (4), $\omega$ is the rotational frequency of the rotating element and $\mathrm{P}$ is the azimuthal angle of the fixed input polarizer. Equation (4) shows that the RAE detector system does not directly measure the ellipsometric parameters $\Psi$ and $\Delta$, but instead normalized Fourier coefficients $\alpha$ and $\beta$. Equation (5) is used to transform the $\alpha$ and $\beta$ measured by an RAE into ellipsometric parameters $\Psi$ and $\Delta$.

$$
\begin{gathered}
\mathrm{I}(\mathrm{t}) \propto 1+\alpha \cos (2 \omega \mathrm{t})+\beta \sin (2 \omega \mathrm{t}), \quad \alpha=\frac{|R p|^{2} \cos ^{2} P-|R s|^{2} \sin ^{2} P}{|R p|^{2} \cos ^{2} P+|R s|^{2} \sin ^{2} P} \quad \beta=\frac{2 \operatorname{Re}\left(R p \cdot R s^{*}\right) \cos P \sin P}{|R p|^{2} \cos ^{2} P+|R|^{2} \sin ^{2} P} \\
\tan \Psi=\sqrt{\frac{1+\alpha}{1-\alpha}} \tan P \quad \cos \Delta=\frac{\beta}{\sqrt{1-\alpha^{2}}}
\end{gathered}
$$

It is therefore not valid to calculate $\Psi_{\text {eff }}$ and $\Delta_{\text {eff }}$ for the partially polarized beam by simply averaging together the predicted $\Psi$ and $\Delta$ values (or $\tan \Psi$ and $\cos \Delta$ values) for the individual collected beams. The collected beams must be averaged in terms of the measured detector intensity. From inspection of equation (4), it can be concluded that the important quantities to average the multiple beams at the detector are: $(|\mathrm{Rp}|)^{2},(|\mathrm{Rs}|)^{2}$, and $\operatorname{Re}\left(\mathrm{Rp}^{*} \mathrm{Rs}^{*}\right)$. The $\operatorname{Re}\left(\mathrm{Rp} \cdot \mathrm{Rs}^{*}\right)$ term is the real part of the quantity Rp times the complex conjugate of Rs. The effective ellipsometric parameters $\Psi_{\text {eff }}$ and $\Delta_{\text {eff }}$ for the partially polarized beam can then be calculated from equations (6) and (7). In these equations, only the terms representing beams that are collected by the detector are included in the summations.

$$
\begin{aligned}
& \tan \Psi_{\text {eff }}=\sqrt{\frac{\Sigma|R p|^{2}}{\Sigma|R s|^{2}}} \\
& \cos \Delta_{\text {eff }}=\frac{\Sigma \operatorname{Re}\left(R p \cdot R s^{*}\right)}{\sqrt{\Sigma|R p|^{2} \cdot \Sigma|R s|^{2}}}
\end{aligned}
$$

Equations (6) and (7) enable accurate modeling and analysis of RAE or RPE data measured on transparent substrates with incoherent reflections. Similar results have been presented elsewhere ${ }^{8}$, but the current treatment is more general and easily_extended to other ellipsometric measurement situations in which the sample partially depolarizes the measurement beam. To perform a model calculation for a thin film stack on a transparent substrate, first calculate the overall Rp and Rs (or Tp and Ts in the transmission case) for each collected beam by evaluating the Fresnel coefficients and expressions shown in figure 1, for both $\mathrm{p}$ - and s- polarized light. Then use equations (6) and (7) to sum the collected beam components and calculate the effective ellipsometric parameters $\Psi_{\text {eff }}$ and $\Delta_{\text {eff }}$ measured by a rotating element ellipsometer. In practice, it is difficult to accurately predict the number of beams collected by the detector; this uncertainly is handled by simply defining the number of backreflections as a fitting parameter in the model. The expressions shown in figure 1 are also valid for films on the backside of the substrate, as the $\mathrm{Rb}$ and $\mathrm{Tb}$ Fresnel coefficients describing the backside substrate/ambient interface can also represent the overall complex amplitude reflectivity and transmissivity of a multilayer stack.

Figure 2 summarizes the different ellipsometric data types that can be experimentally measured on a sample with a transparent substrate: (a) standard VASE data, (b) reflection ellipsometry with backside reflections, (c) ellipsometry with the sample flipped over, and (d) transmission ellipsometry. This figure also illustrates how the detector system may collect only a limited number, or even a fractional number, of the reflected or transmitted beams. These data types can all be accurately modeled using the formalism described in this section. 


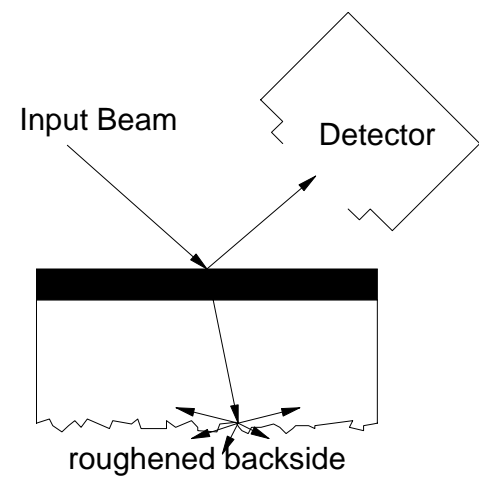

(a) standard VASE data

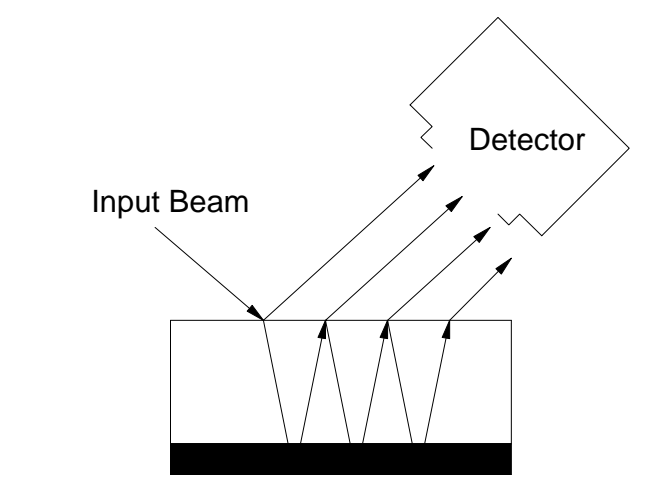

(c) reflection ellipsometry with sample flipped over

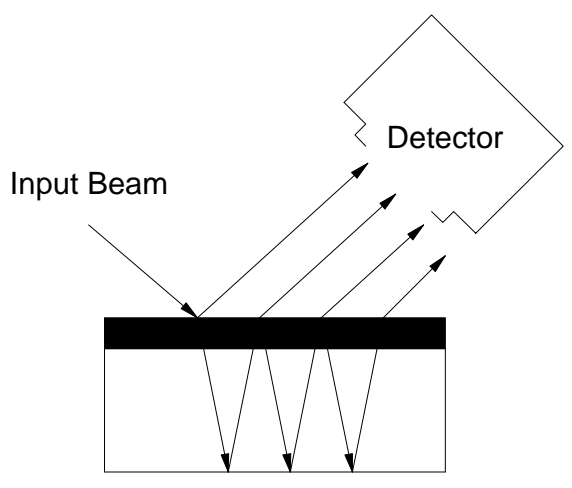

(b) reflection ellipsometry with backside reflections

(d) transmission ellipsometry

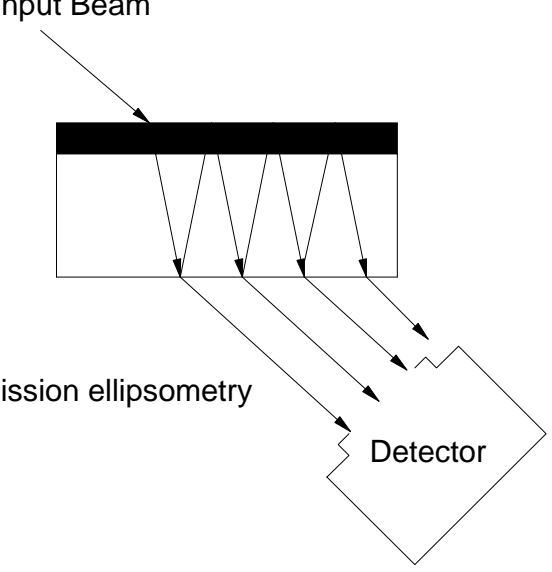

Figure 2. Ellipsometric data types acquired on sample with transparent substrate.

\section{ANALYSIS EXAMPLE: Cr-BASED PHASE-SHIFTING PHOTOMASK BLANKS}

Attenuated phase-shifting photomasks offer improved lithographic printing performance. The Cr-based phaseshifting photomask blank analyzed in this section was produced by batch inline reactive sputtering. The details of the photomask blank fabrication and testing are given elsewhere. An ideal phase-shifting photomask blank should have a transmitted phase shift, relative to air, of $180^{\circ}$. In addition, a number of other important mask properties must be optimized and controlled, including transmitted and reflected beam intensity, conductivity, etchability, and compatibility with existing aligners. A graded film structure provides the photomask blank designer the degrees of freedom necessary to simultaneously optimize for all of the desired properties. However, optical characterization of a complex graded, absorbing structure is very difficult, and can only be successfully achieved by a combined analysis using multiple optical data types. 
Table 1 lists the optical data types that were experimentally measured to analyze the complicated phase-shifter structure. While VASE data was acquired at 4 angles of incidence, only the $75^{\circ}$ data was used in the fit. Data for the other ellipsometric data types, which all included incoherent substrate reflection effects, was also acquired only at $75^{\circ}$. This simplified the data analysis, as the number beams collected by the detector could be assumed constant for all of the measured data types. The multiple optical data types help to overdetermine the model in the data analysis. The ellipsometric data from the reverse direction was especially useful, as it provided information about the substrate/film interface, while most of the other measurements are more sensitive to the ambient/film interface.

\begin{tabular}{|c|c|l|}
\hline Data Type & Angle of Incidence & Description \\
\hline $\mathrm{E}$ & $60-75$ by 5 & standard VASE data: reflection ellipsometric data with backside roughened \\
\hline $\mathrm{Eb}$ & 75 & reflection ellipsometric data with backside reflections \\
\hline $\mathrm{Er}$ & 75 & reflection ellipsometric data with the sample flipped over \\
\hline $\mathrm{Et}$ & 75 & ellipsometric transmission data \\
\hline $\mathrm{pT}$ & 0 & intensity transmission data \\
\hline
\end{tabular}

Table 1. Optical data types measured on the Cr-based phase-shifter sample.

To analyze the sample, a model representing the graded structure of the Cr-based phase-shifter film was first constructed. Tabulated $\mathrm{SiO}_{2}$ optical constants were used to model the fused silica substrate. A single graded film, with a nominal thickness determined by stylus profilometry of $122.5 \mathrm{~nm}$, was used to represent the Cr-based phase-shifter film. Sputtered Neutrals Mass Spectrometry (SNMS) data, which is shown in figure 4, and Rutherford Backscattering Spectrometry (RBS) suggested a triangular profile for the index gradient in the film. Near the middle of the film, the oxygen content is lower, and the optical constants should exhibit a more metallic behavior. At the film surface and substrate interface, the higher oxygen content should lead to more dielectric-like optical constants. It was assumed that the optical constants at any depth in the film could be described by a mixture of metallic-like and dielectric-like optical constants. The Bruggeman Effective Medium Approximation (EMA) was used to describe the resultant optical constants of the two constituent mixture. The index gradient in the film was parameterized by allowing the EMA fraction of the dielectric-like constituent to vary as a function of depth. The dielectric-like EMA fraction was $100 \%$ at the film surface, $0 \%$ at the middle node, and some unknown intermediate value at the film/substrate interface. To numerically calculate the graded optical model, the analysis software automatically sliced the graded layer into 10 sublayers, and calculated the EMA fraction for each sublayer to maintain a triangular profile, as shown in figure 4. The fitting parameters used characterize the model structure were: 1) total film thickness, 2) middle node position of the peak in the triangular profile, 3) the EMA fraction at the film/substrate interface, and 4) the number of backreflections collected by the detector.

To reduce the number of fitting parameters, the optical constants of the two constituent materials were each parameterized by the simple polynomial dispersion functions shown in equation (8). Absolutely no prior information was available about the optical constants of the constituent materials, except that one of the materials should be more metallic, and the other more dielectric. The polynomial dispersion functions allowed enough flexibility in the model to represent optical constants from metallic, to dielectric, or anywhere inbetween, while still constraining the optical constants to smooth, continuous curves. Without prior knowledge, it was not possible to use more conventional dispersion formulas (Cauchy, Sellmeier, Lorentz oscillators, etc.), which may not be applicable to the absorbing, amorphous, non-stoichiometric constituent materials found in the Cr-based phase-shifting film.

$$
\mathrm{n}(\lambda)=\mathrm{A}_{\mathrm{n}}+\mathrm{B}_{\mathrm{n}} \cdot \lambda+\mathrm{C}_{\mathrm{n}} \cdot \lambda^{2}+\mathrm{D}_{\mathrm{n}} \cdot \lambda^{3}+\mathrm{E}_{\mathrm{n}} \cdot \lambda^{4}, \quad \mathrm{k}(\lambda)=\mathrm{A}_{\mathrm{k}}+\mathrm{B}_{\mathrm{k}} \cdot \lambda+\mathrm{C}_{\mathrm{k}} \cdot \lambda^{2}+\mathrm{D}_{\mathrm{k}} \cdot \lambda^{3}+\mathrm{E}_{\mathrm{k}} \cdot \lambda^{4}, \quad \lambda \text { in } \mu \mathrm{m}
$$

The experimental data fits resulting from the Levenberg-Marquardt regression analysis are shown in figure 5 . Excellent agreement between the model fit data (solid lines) and experimentally measured data (dashed lines) is observed for all data types. The MSE for the fit (see equation 1) was 2.36. The model fit structural parameters are summarized in table 2. The error bars on the fit parameters are single parameter $90 \%$ confidence limits. The optical constants extracted for the two constituent materials are shown in figure 3. The depth profile of the graded model is compared with the SNMS profile in figure 4 (the $\mathrm{x}$-axis scales for the two profiles are reversed with respect to each other). 


\begin{tabular}{|l|l|}
\hline Fit Parameter Description & Best Fit Value \\
\hline Total Film Thickness & $1199 \pm 3.7 \AA$ \\
\hline \begin{tabular}{l} 
Grading Node $\begin{array}{c}\text { ( } 0 \% \equiv \text { film/substrate interface, } \\
\text { Relative Position } \quad 100 \% \equiv \text { ambient/film interface })\end{array}$ \\
\hline EMA fraction at film/substrate interface
\end{tabular} & $36.8 \pm 0.43 \%$ \\
\hline Number of backside reflections collected by detector & $1.90 \pm 0.014$ \\
\hline
\end{tabular}

Table 2. Best fit model structural parameters for Cr-based phase-shifting graded film.

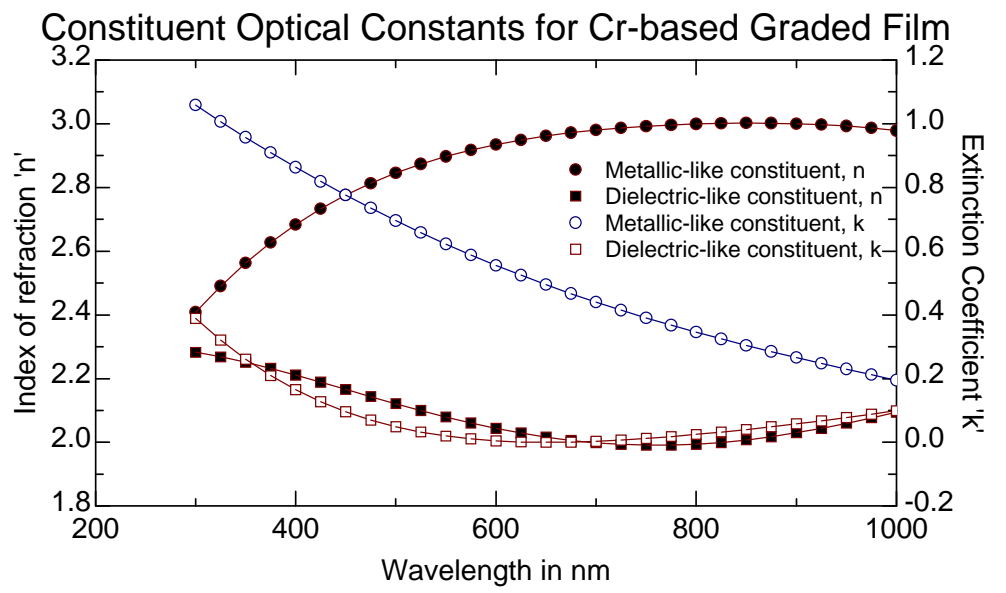

Figure 3. Extracted constituent optical constants for the Cr-based phase-shifting photomask sample.
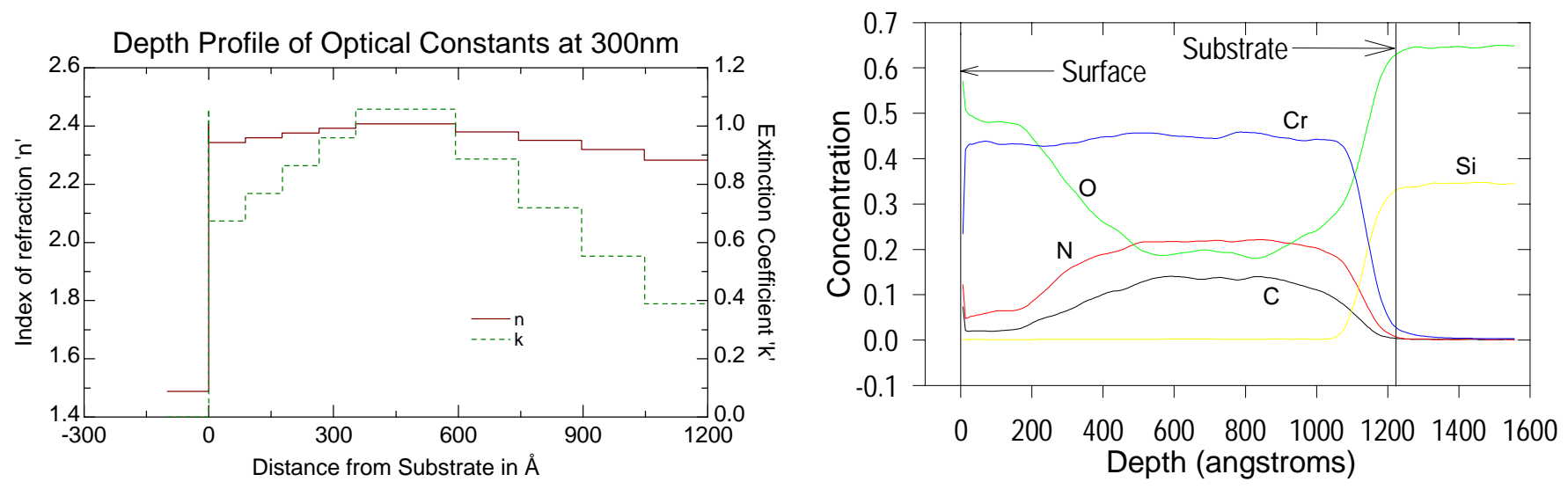

Figure 4. Ellipsometrically determined depth profile compared with the SNMS profile 

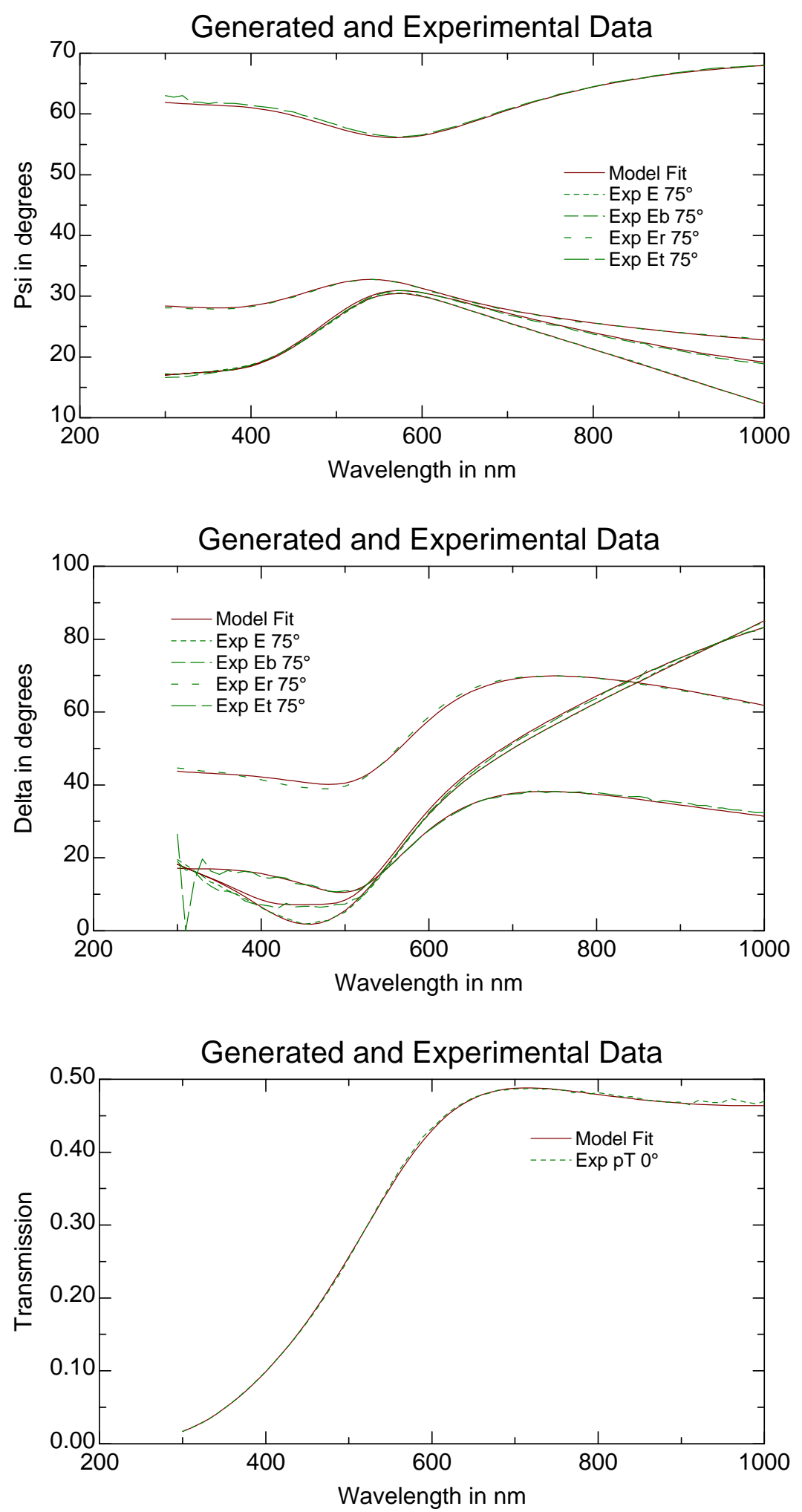

Figure 5. Data fits for Cr-based phase-shifting photomask sample to the multiple optical data types. 
The most important parameter for a phase-shifting photomask is its transmitted phase shift relative to air; this parameter is also highly sensitive to the internal structure of the film. Figure 6 compares the transmitted relative phase shift calculated from the best fit optical model with the experimentally measured values at 3 laser wavelengths. The close agreement confirms that the model structure and optical constants (determined using the multiple optical data types, but without the transmitted phase shift information) are physically reasonable, and therefore useful for designing subsequent $\mathrm{Cr}$ based graded phase-shifting films.

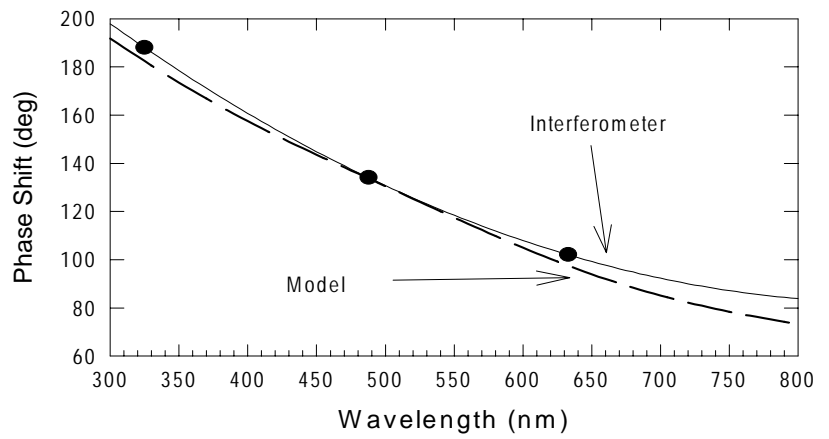

Figure 6. Spectral transmitted phase shifts determined by the optical model and the phase sensitive interferometer

\section{$\underline{\text { 5. DISCUSSION }}$}

The detailed optical model extracted from the multiple optical data type analysis is extremely important in understanding the complex optical behavior of the phase-shifting photomask blank. However, even with the multiple optical data types, fitting for two sets of optical constants and the grading structure in this complex film is pushing the limits of this analysis technique. Parameterizing the optical constants of the constituent materials with a more physical dispersion model may help to constrain the fit. A improved data fitting algorithm that combines the Levenberg-Marquardt method with simulated annealing and/or singular value decomposition may also help the analysis software converge more reliably to the best possible data fit.

\section{ACKNOWLEDGMENTS}

BJ would like to thank Robert Edgerton for many insightful discussions concerning the polarization and coherence properties of light beams. RHF would like to thank Catherine Wilson for materials modeling.

\section{REFERENCES}

${ }^{1}$ J.A. Woollam and P.G. Snyder, "Variable Angle Spectroscopic Ellipsometry", book chapter for Encyclopedia of Materials Characterization, Butterworth Publishers, Greenwich, 1992.

${ }^{2}$ William A. McGahan, Blaine Johs, and John A. Woollam, "Techniques for ellipsometric measurement of the thickness and optical constants of thin absorbing films", Thin Solid Films, vol. 234, pp. 443-446, 1993.

${ }^{3}$ R.M.A. Azzam and N.M. Bashara, Ellipsometry and Polarized Light, North-Holland, Amsterdam, 1977.

${ }^{4}$ W.H. Press et.al., Numerical Recipes in C, Cambridge University Press, Cambridge, 1988.

${ }^{5}$ J.A. Woollam Co., Lincoln, NE USA.

${ }^{6}$ Roy F. Potter, "Basic Parameters for Measuring Optical Properties", in Handbook of Optical Constants of Solids, edited by Edward D. Palik, Academic Press, Orlando, 1985.

${ }^{7}$ R.W. Collins, "Automatic rotating element ellipsometers: Calibration, operation, and real-time applications", Rev. Sci. Instrum, vol. 61(8), pp. 2029-2062, 1990.

${ }^{8}$ J.T.H. Zettler and L. Schrottke, "Ellipsometric Characterization of Layers on Transparent Semiconductor Wafers", Physica Status Solidi (b), vol. 163, pp. 69-74, 1991.

${ }^{9}$ for example, non-uniform film thickness or finite bandwidth of the measurement beam

${ }^{10}$ F.D. Kalk, R.H. French, H.U. Alpay, and G. Hughes, "Attenuated phase-shifting photomasks fabricated from Cr-based embedded shifter blanks", SPIE proceedings from Photomask Japan 1994 (to be published).

${ }^{11}$ D.E. Aspnes, "The accurate determination of optical properties by ellipsometry", in Handbook of Optical Constants of Solids, edited by Edward D. Palik, Academic Press, Orlando, 1985. 
B. Johs, R. H. French, F. D. Kalk, W. A. McGahan, J. A. Woollam, "Optical Analysis of Complex Multilayer Structures Using Multiple Data Types", submitted to SPIE Proceedings on Optical Interference Coatings, Grenoble France, June 1994.

B. Johs, R. H. French, F. D. Kalk, W. A. McGahan, J. A. Woollam, "Optical Analysis of Complex Multilayer Structures Using Multiple Data Types", Optical Interference Coatings, SPIE Vol. 2253, Edited by F. Abeles, 1098-1106, (1994). 